\title{
ON A DOUBLY MIXED BOUNDARY VALUE PROBLEM FOR AN ELASTIC LAYER*
}

By R. D. LOW (Department of Mathematics, University of Michigan)

1. Introduction. Consider an infinite, isotropic, homogeneous elastic layer included between the planes $z=0$ and $z=h$ of a cylindrical coordinate system $(r, \theta, z)$. The $z$-axis is directed downward and the plane $z=h$ with the circular portion $0 \leq r \leq 1$ deleted is taken as a rigid foundation upon which the layer rests. A rigid, flat-ended circular punch of unit radius is pressed into the upper boundary $z=0$ to a depth $\epsilon$ by the application of an axial force $P$. Frictional forces exerted by the punch and the foundation on the boundaries of the layer are neglected, as are the body forces. Thus the problem is to determine the solution of Navier's equation

$$
\mu \nabla^{2} \mathbf{u}+(\lambda+\mu) \nabla(\nabla \cdot \mathfrak{u})=\mathbf{0},
$$

for the displacement vector $\mathbf{u}=\left(u_{r}, 0, u_{z}\right)$ subject to the boundary conditions

$$
\begin{gathered}
\sigma_{r z}(r, 0)=\sigma_{r z}(r, h)=0, \quad 0 \leq r<\infty, \\
u_{z}(r, 0)=\epsilon, \quad 0 \leq r \leq 1, \\
u_{z}(r, h)=0, \quad 1 \leq r<\infty, \\
\sigma_{z z}(r, 0)=0, \quad 1<r<\infty, \\
\sigma_{z z}(r, h)=0, \quad 0 \leq r<1,
\end{gathered}
$$

and

$$
\text { \{all stress and displacement components vanish as } r \rightarrow \infty \text { \}. }
$$

In the above, $\lambda$ and $\mu$ are the Lamé constants; $\sigma_{z z}$ and $\sigma_{r z}$ are the normal and shearing stress components respectively.

2. Reduction to a system of Fredholm integral equations. It is known [1] that

$$
2 \mu \mathbf{u}=\nabla F+z \nabla G-(3-4 \sigma) G \mathbf{k}
$$

is a solution of (1) where $F(r, z)$ and $G(r, z)$ are harmonic, $\sigma$ is Poisson's ratio, and $\mathbf{k}$ is the unit vector in the positive $z$-direction. From (8) it follows that the elastic quantities appearing in the boundary conditions (2) through (6) are given in terms of $F$ and $G$ by

$$
\begin{aligned}
2 \mu u_{z}(r, z) & =F_{z}+z G_{z}-(3-4 \sigma) G, \\
\sigma_{z s}(r, z) & =F_{z z}+z G_{z z}-2(1-\sigma) G_{z}, \\
\sigma_{r z}(r, z) & =F_{r z}+z G_{r z}-(1-2 \sigma) G_{r},
\end{aligned}
$$

where the subscripts on $F$ and $G$ denote partial derivatives. We take for $F$ and $G$ the following integral representations:

$$
\begin{aligned}
& F(r, z)=\int_{0}^{\infty}[A \cosh \alpha z+B \sinh \alpha z] J_{0}(\alpha r) d \alpha \\
& G(r, z)=\int_{0}^{\infty}[C \cosh \alpha z+D \sinh \alpha z] J_{0}(\alpha r) d \alpha
\end{aligned}
$$

*Received August 22, 1963; revised manuscript received October 28, 1963. 
in which $A, B, C$, and $D$ are functions of $\alpha$ to be determined by the boundary conditions.

Since both of (2) are to hold for all $r \geq 0$, their application requires that $\alpha B=(1-2 \sigma) C$ and $\alpha A+\alpha h C+(\alpha h \operatorname{coth} \alpha h-1+2 \sigma) D=0$. If we now impose the boundary conditions (3) through (6) and use the above results to eliminate $A$ and $B$, the following system of integral equations is obtained:

$$
\begin{gathered}
\int_{0}^{\infty} C J_{0}(\alpha r) d \alpha=-\mu \epsilon /(1-\sigma), \quad 0 \leq r \leq 1, \\
\int_{0}^{\infty}[C \cosh \alpha h+D \sinh \alpha h] J_{0}(\alpha r) d \alpha=0, \quad 1 \leq r<\infty, \\
\int_{0}^{\infty}[\alpha h C+(1+\alpha h \operatorname{coth} \alpha h) D] \alpha J_{0}(\alpha r) d \alpha=0, \quad 1<r<\infty, \\
\int_{0}^{\infty}\left[C \sinh \alpha h+\frac{\alpha h+\sinh \alpha h \cosh \alpha h}{\sinh \alpha h} D\right] \alpha J_{0}(\alpha r) d \alpha=0, \quad 0 \leq r<1 .
\end{gathered}
$$

Thus all conditions will be satisfied if we can determine the functions $C$ and $D$ from what might be called: the set of "quadruple integral equations" (14) through (17).

The following reduction of this quadruple set to a system of two Fredholm integral equations is a repeated application of a technique employed by Lebedev and Uflyand [2] to reduce a pair of dual integral equations to a single Fredholm equation. Hence if we introduce the new unknown functions $\phi(t)$ and $\psi(t)$ and define

$$
\begin{gathered}
C \cosh \alpha h+D \sinh \alpha h=\int_{0}^{1} \phi(t) \sin \alpha t d t, \quad \phi(0)=0, \\
\alpha h C+(1+\alpha h \operatorname{coth} \alpha h) D=\int_{0}^{1} \psi(t) \cos \alpha t d t,
\end{gathered}
$$

then it is easily shown that (15) and (16) are satisfied identically. It is then a simple matter to determine $C$ and the combination $C \sinh \alpha h+(\alpha h+\sinh \alpha h \cosh \alpha h) D / \sinh \alpha h$ in terms of $\phi$ and $\psi$. The results are respectively

and

$$
\left[-1+g_{1}(\alpha h)\right] \int_{0}^{1} \psi(t) \cos \alpha t d t+g_{2}(\alpha h) \int_{0}^{1} \phi(t) \sin \alpha t d t
$$

$$
g_{2}(\alpha h) \int_{0}^{1} \psi(t) \cos \alpha t d t+\left[1-g_{3}(\alpha h)\right] \int_{0}^{1} \phi(t) \sin \alpha t d t,
$$

where $g_{1}(\lambda)=\left(\lambda+e^{-\lambda} \sinh \lambda\right) / E(\lambda), g_{2}(\lambda)=(\sinh \lambda+\lambda \cosh \lambda) / E(\lambda), g_{3}(\lambda)=\left(\lambda^{2}+\lambda+\right.$ $\left.e^{-\lambda} \sinh \lambda\right) / E(\lambda)$, and $E(\lambda)=\lambda+\sinh \lambda \cosh \lambda$. If we now insert the above into (14) and (15), make use of the result [3]

$$
\int_{0}^{\infty} J_{0}(\alpha r) \cos \alpha t d \alpha=\left\{\begin{array}{cc}
\left(r^{2}-t^{2}\right)^{-1 / 2}, & r>t, \\
0 & r<t,
\end{array}\right.
$$

and replace $J_{0}(\alpha r)$ by its Poisson integral representation, we find

$$
\begin{aligned}
\int_{0}^{r} \frac{d x}{\sqrt{r^{2}-x^{2}}}\left[\psi(x)-\int_{0}^{1} \psi(t)\left\{G_{1}(x+t)+G_{1}(x-t)\right\} d t\right. \\
\left.\quad-\int_{0}^{1} \phi(t)\left\{G_{2}(x+t)-G_{2}(x+t)\right\} d t\right]=\mu \epsilon /(1-\sigma), \quad 0 \leq r \leq 1,
\end{aligned}
$$


and

$$
\begin{aligned}
\int_{0}^{r} \frac{d x}{\sqrt{r^{2}-x^{2}}}\left[\phi^{\prime}(x)\right. & +\int_{0}^{1} \psi(t)\left\{G_{2}^{\prime}(x+t)+G_{2}^{\prime}(x-t)\right\} d t \\
& \left.+\int_{0}^{1} \phi(t)\left\{G_{3}^{\prime}(x+t)-G_{3}^{\prime}(x-t)\right\} d t\right]=0, \quad 0 \leq r<1,
\end{aligned}
$$

where

$$
G_{1,3}(u)=\int_{0}^{\infty} g_{1,3}(\alpha h) \cos \alpha u d \alpha, \quad G_{2}(u)=\int_{0}^{\infty} g_{2}(\alpha h) \sin \alpha u d \alpha .
$$

Equations (18) and (19) are Abel type integral equations from which we obtain the following system of Fredholm integral equations:

$$
\begin{array}{ll}
\psi(x)-\int_{0}^{1} K_{1}(x, t) \psi(t) d t-\int_{0}^{1} K_{2}(x, t) \phi(t) d t=\beta, & 0 \leq x \leq 1, \\
\phi(x)+\int_{0}^{1} K_{3}(x, t) \psi(t) d t+\int_{0}^{1} K_{4}(x, t) \phi(t) d t=0, & 0 \leq x<1,
\end{array}
$$

where $K_{1}, K_{2}, K_{3}$, and $K_{4}$ are given respectively by $G_{1}(x+t)+G_{1}(x-t), G_{2}(x+t)-$ $G_{2}(x-t), G_{2}(x+t)+G_{2}(x-t)$, and $G_{3}(x+t)-G_{3}(x-t)$, and $\beta=2 \mu \epsilon / \pi(1-\sigma)$. In obtaining (21) from the solution of (19), we have integrated with respect to $x$ from zero to $x$, used $\phi(0)=0$, and the fact that $G_{2}(u)$ is odd and $G_{3}(u)$ is even.

Due to the nature of the kernels, the solution of the above system will have to be obtained numerically if $h$ is small compared to unity, but an approximate solution can be found by the usual iteration methods when $h$ is large. Such a solution, correct to $0\left(h^{-4}\right)$, will be determined in section 4 below.

3. Boundary values expressed in terms of $\phi$ and $\psi$. In this section we show that, on those portions of the boundary where they are not prescribed, the boundary values $u_{z}(r, h), \sigma_{z z}(r, 0), u_{z}(r, 0)$, and $\sigma_{z z}(r, h)$ are directly expressible in terms of $\phi$ and $\psi$, thus making it unnecessary to determine the function $A, \cdots, D$. Except for the constant factor $-(1-\sigma) / \mu$, the left side of $(15)$ is equal to $u_{z}(r, h)$ for all $r$. In particular when $0 \leq r \leq 1$, we find

$$
\mu u_{z}(r, h)=-(1-\sigma) \int_{r}^{1} \frac{\phi(t) d t}{\sqrt{t^{2}-r^{2}}}
$$

In a similar manner, we obtain from (16) when $0 \leq r<1$

$$
\sigma_{z z}(r, 0)=-\psi(1)\left(1-r^{2}\right)^{-1 / 2}+\int_{r}^{1} \frac{\psi^{\prime}(t) d t}{\sqrt{t^{2}-r^{2}}}
$$

Incidentally, this last result relates the axial force $P$ and the function $\psi$. We have

$$
P=-2 \pi \int_{0}^{1} \sigma_{z z}(r, 0) r d r=2 \pi \int_{0}^{1} \psi(t) d t .
$$

The remaining boundary values are not so simply expressed, but it is not difficult to show, using (20) and (21), that

$$
\begin{aligned}
& \mu u_{z}(r, 0)=(2 \mu \epsilon / \pi) \sin ^{-1}(1 / r)-(1-\sigma) \int_{1}^{r} \frac{d x}{\sqrt{r^{2}-x^{2}}} \int_{0}^{1}\left[K_{1}(x, t) \psi(t)\right. \\
&\left.+K_{2}(x, t) \phi(t)\right] d t, \quad 1 \leq r<\infty,
\end{aligned}
$$


and

$$
\begin{aligned}
\sigma_{s s}(r, h)=\phi(1)\left(r^{2}-1\right)^{-1 / 2}-\int_{1}^{r} \frac{d x}{\sqrt{r^{2}-x^{2}}} \frac{d}{d x} \int_{0}^{1}[ & K_{3}(x, t) \psi(t) \\
& \left.\quad+K_{4}(x, t) \phi(t)\right] d t, \quad 1<r<\infty .
\end{aligned}
$$

This last result furnishes a necessary condition for the validity of the solution of the posed problem; namely, $\sigma_{z z}(r, h) \leq 0$ when $r>1$. For otherwise the layer would break contact with the foundation and $u_{z}(r, h)$ would not be zero as required in boundary condition (4).

Finally, it is perhaps worth noting that the solution of the problem of a punch acting on a half space follows from the above in the limit as $h \rightarrow \infty$. For in this case the kernels $K_{i}$ tend to zero and the solution of the system (20) and (21) is simply $\psi(x)=\beta, \phi(x)=0$. Then the previous expressions give $u_{z}(r, 0)=(2 \epsilon / \pi) \sin ^{-1}(1 / r), 1 \leq r<\infty ; \sigma_{z z}(r, 0)=$ $-\beta\left(1-r^{2}\right)^{-1 / 2}, 0 \leq r<1$; and $P=4 \mu \epsilon /(1-\sigma)$. These are, of course, well known, and hence serve as a partial check on the method of solution.

4. Approximate solution for large $h$. In this section we obtain the solution of the system (20), (21) correct to $0\left(h^{-4}\right)$ by the usual iteration process. We take as the zeroth approximation $\psi_{0}(x)=\beta, \phi_{0}(x)=0$, and then the $n$th approximation is to be determined from

$$
\begin{aligned}
& \psi_{n}(x)=\beta+\int_{0}^{1} K_{1}(x, t) \psi_{n-1}(t) d t+\int_{0}^{1} K_{2}(x, t) \phi_{n-1}(t) d t \\
& \phi_{n}(x)=-\int_{0}^{1} K_{3}(x, t) \psi_{n-1}(t) d t-\int_{0}^{1} K_{4}(x, t) \phi_{n-1}(t) d t, \quad n=1,2,3, \cdots .
\end{aligned}
$$

If the kernels $K_{i}$ are expanded in decreasing powers of $h$ and powers of $h^{-1}$ higher than the fourth are discarded, we obtain

$$
\begin{aligned}
& K_{1}(x, t)=\frac{A_{0}}{h}-\frac{A_{1} t^{2}}{2 h^{3}}-\frac{A_{1}}{2 h^{3}} x^{2}+0\left(h^{-5}\right), \\
& K_{2}(x, t)=\frac{B_{0} t}{h^{2}}-\frac{B_{1} t^{3}}{6 h^{3}}-\frac{B_{1} t}{2 h^{4}} x^{2}+0\left(h^{-6}\right), \\
& K_{3}(x, t)=\left(\frac{B_{0}}{h^{2}}-\frac{B_{1} t^{2}}{2 h^{4}}\right) x-\frac{B_{1}}{6 h^{4}} x^{3}+0\left(h^{-6}\right), \\
& K_{4}(x, t)=-\frac{c_{0} t}{h^{3}} x+0\left(h^{-5}\right),
\end{aligned}
$$

where

$$
\begin{gathered}
\pi A_{n}=2 \int_{0}^{\infty} \lambda^{2 n} g_{1}(\lambda) d \lambda, \quad \pi B_{n}=2 \int_{0}^{\infty} \lambda^{2 n+1} g_{2}(\lambda) d \lambda, \\
\pi C_{n}=2 \int_{0}^{\infty} \lambda^{2 n+2} g_{3}(\lambda) d \lambda .
\end{gathered}
$$

With the above truncated kernels it is found that four iterations are sufficient to give 


$$
\begin{gathered}
\psi(x)=\beta\left(a+b x^{2}\right)+0\left(h^{-5}\right), \phi(x)=-\beta\left(c x+d x^{3}\right)+0\left(h^{-5}\right), \text { where } \\
a=1+\frac{A_{0}}{h}+\frac{A_{0}^{2}}{h^{2}}+\frac{6 A_{0}^{3}-A_{1}}{6 h^{3}}+\frac{6 A_{0}^{4}-3 A_{0} A_{1}-2 B_{0}^{2}}{6 h^{4}}, \\
b=-\frac{A_{1}}{2 h^{3}}-\frac{A_{0} A_{1}}{2 h^{4}}, \quad c=\frac{B_{0}}{h^{2}}+\frac{A_{0} B_{0}}{h^{2}}+\frac{6 A_{0}^{2} B_{0}-B_{1}}{6 h^{4}}, \quad d=-\frac{B_{1}}{6 h^{4}} .
\end{gathered}
$$

It is then a simple matter to obtain

$$
\begin{array}{cc}
u_{z}(r, h)=(2 \epsilon / \pi)\left(1-r^{2}\right)^{1 / 2}\left[c+d\left(4 r^{2}-1\right) / 3\right]+0\left(h^{-5}\right), & 0 \leq r \leq 1, \\
\sigma_{z z}(r, 0)=-\beta\left(1-r^{2}\right)^{-1 / 2}\left(a-b+2 b r^{2}\right)+0\left(h^{-5}\right), & 0 \leq r<1 .
\end{array}
$$

It is interesting to note from the above expression for $u_{z}(r, h)$ that since $c=0\left(h^{-2}\right)$, the effect of the hole in the foundation is negligible when $h$ is so large that terms of order $h^{-2}$ can be ignored. For in this case $u_{z}(r, h)$ would be zero for all $r$, which is the boundary condition one would use in place of (4) and (6) when the foundation contains no hole.

\section{REFERENCES}

[1] A. E. Green and W. Zerna, Theoretical elasticity, Clarenden Press, Oxford, 1954

[2] N. N. Lebedev and Ya. S. Uflyand, Axisymmetric contact problem for an elastic layer, J. Appl. Math. Mech. 22 (1958) 442 [English translation of Prik. Mat. i Mech., 22 (1958) 320]

[3] W. Magnus and F. Oberhettinger, Formulas and Theorems for the special functions of mathematical physics, Chelsea, New York, 1949

\section{ON THE OSCILLATIONS OF A PENDULUM UNDER PARAMETRIC EXCITATION*}

By R. A. STRUBLE (University of North Carolina, Raleigh, N. C.)

In a recent paper [1], we examined the oscillations of a pendulum under parametric excitation using a formal asymptotic method. It is the purpose of this note to point out that the general behavior suggested for this system may be corroborated through the application of a new mapping theorem due to Moser [2].

Consider the nonlinear equation

$$
\frac{d^{2} \theta}{d t^{2}}+\left(\omega_{0}^{2}-\frac{\xi_{0} \omega^{2}}{L} \cos \omega t\right) \sin \theta=0
$$

where $\omega, \omega_{0}, \xi_{0}$ and $L$ are positive constants with $\xi_{0} / L$ small. This equation depicts the motion of a simple pendulum which is excited parametrically by small, vertical vibrations of its support. (See [1]). The free motions for $\xi_{0}=0$ are well known [3] and can be described by the energy integral

$$
\frac{1}{2}\left(\frac{d \theta}{d t}\right)^{2}-\omega_{0}^{2} \cos \theta=E .
$$

*Received Sept. 5, 1963; revised manuscript received October 25, 1963. Work sponsored by the U. S. Army Research Office (Durham). 